\title{
AC 2007-1697: TECHNOLOGIES TO INTRODUCE EMBEDDED DESIGN EARLY IN ENGINEERING
}

Shekhar Sharad, National Instruments 


\title{
Technologies to Introduce Embedded Design Early in Engineering
}

\author{
Shekhar Sharad \\ Academic Product Manager \\ National Instruments \\ shekhar.sharad@ni.com
}

\section{Introduction}

With decreasing cost of silicon and increasing demand on features, almost every device around us is becoming "embedded"[1] - from cell phones to irons, from cars to GPS units. Embedded devices are being used in every industry, in aerospace, mechanical, chemical and even biomedical engineering. That said, there is a need to create excitement and awareness about this exciting and pervasive field to the students freshly enrolled in engineering because no matter which major they choose, they will eventually have to deal with embedded devices. It is also widely acknowledged that embedded programming is not trivial and hence may not be the most appropriate form to be introduced early in the engineering career; hence, there is a continual search for better tools and techniques to teach with embedded devices early in engineering. One of the more promising methodologies available today is Graphical Programming.

\section{Why Embedded Devices Early?}

Given the programming complexity for embedded devices, it warrants the question, "Why Embedded Devices early in engineering? Why can't some other form of instrumentation be introduced?" The reasoning is simple, in order to create excitement in engineering among students today, we must use objects that resonate with them - which they have seen or used in their everyday life. Today, the teenagers see the following devices - MP3 players, cell phones, digital cameras, camcorders etc. These are all embedded devices and almost all of these devices seem to have more and more features being built into them. Hence, it makes it very interesting for the students if they can design/program a hardware platform to play MP3s, or take pictures and process them. As students, their minds resonate with these kinds of platforms and help to get them engaged in engineering which forms a good base to introduce other forms of instrumentation later on in their career

Another key reason for educators is the cost involved. The cost of buying traditional instrumentation is very high. With the introduction of Virtual Instrumentation[2], this has been addressed to a large extent, and embedded devices are the lowest cost devices today. Hence, embedded devices form one of the best platforms to introduce early in engineering because of their cost benefits and their ability to resonate with what the students see in their daily life. This does pose a problem - with the complexity of programming these devices, how do we raise the level of abstraction so that freshmen engineering students can take advantage of these platforms?

\section{Need for Higher Level of Abstraction}

Traditional programming techniques for embedded devices need prospective embedded programmers to know $\mathrm{C}, \mathrm{C}++$ or VHDL in-depth in order to perform bit-level operations. This is a valid approach for optimization purposes. However, most freshman and sophomore engineering students do not have such in-depth knowledge in programming; moreover, the 
concept of embedded programming is alien to most. One of the ways to solve this conundrum is to implement technologies that provide a higher level of abstraction. Such an approach would enable students to focus more on the design and less on the individual bits.

Research[3] indicates that in order to program embedded systems, a major shift in paradigm is required. Literature $[4,5,6]$ shows that the actor-oriented or graphical programming languages are better suited for embedded design because they are based on the dataflow paradigm. Dataflow programming [7] uses the imperative programming model wherein the program is modeled as a series of operations with the data seemingly invisible. While this may seem to be a minor detail, it has a large impact on the applicability of the data flow paradigm to embedded programming. One advantage for example, is that this enables dataflow programming paradigm to be as applicable to multi-core and multi-processor systems as it is to single-core systems. Figure 1 shows some examples of graphical programming languages based on dataflow paradigm.

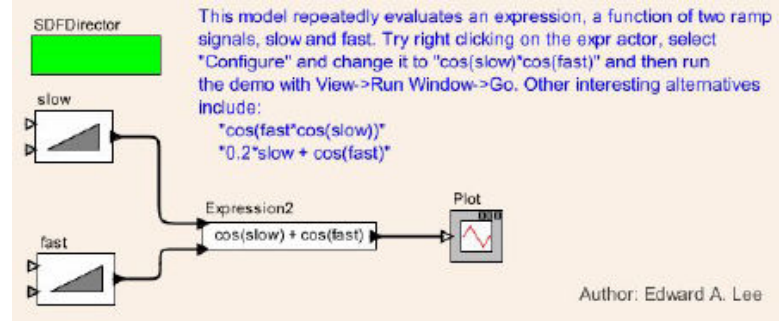

(a) Ptolemy[8]

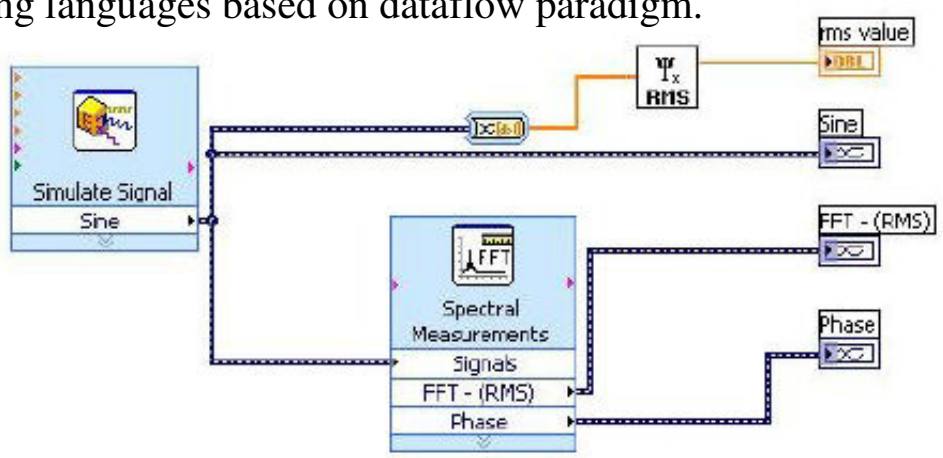

(b) NI LabVIEW[9]

Figure 1. Examples of Graphical Programming Languages based on Data Flow Paradigm

As it can be seen in figure 1, almost all the true graphical programming languages use a block diagram format that coincides with the way engineering students are taught algorithms and designs. This makes it easy for them to translate the theory they learn in class to actual experiments that run on hardware. Graphical programming also presents a "black box" approach to targeting common hardware platforms. As it can be seen in figure 2, embedded design engineers need to go through several steps before they can target a specific hardware. The aim of graphical programming is to provide a single platform that can help achieve this goal by abstracting the details at the implementation level. This helps engineering students who are using embedded devices to quickly design, prototype and deploy an embedded system. 


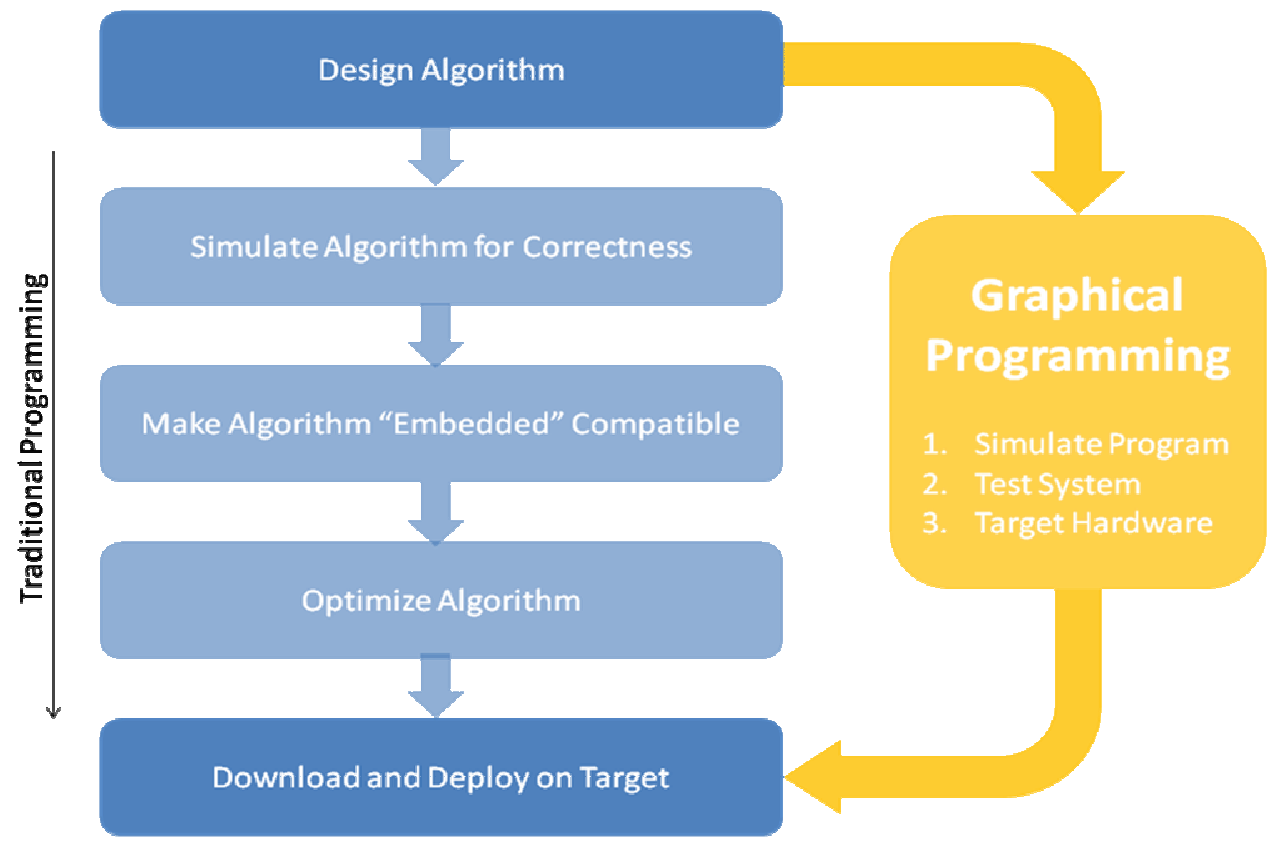

Figure 2. Higher Level of Abstraction with Graphical Programming

\section{Example Implementations with Graphical Programming}

Because of this advantage of higher level of abstraction, graphical programming for embedded systems has turned from a novel idea to a practical implementation possibility. In the following sections we will highlight two such examples, the first example is the use of an embedded device to promote excitement in science and engineering in the high-schools and introductory classes at the University level and the second example is from a senior design project from RPI where a team went from design and simulation to deployment in one semester

\subsection{Use Case: Embedded Devices in the High-school and Freshman levels}

The Infinity Project[10] is an award-winning high school and early college program developed at Southern Methodist University(SMU) in Dallas, TX and is aimed at creating excitement in science and engineering in high school. The aim of the program is to bring math and science to life by using hands-on learning techniques and show the relevance of math and science to other hitech engineering professions thereby increasing interest and enrollment in engineering.

This program is currently implemented in 150 high schools and several universities in the United States and worldwide. The key components of the Infinity Project is an innovative curriculum and textbook written by well-known authors in the field of engineering, the in-depth training offered to all the instructors who want to implement the Infinity Project at their schools and the Infinity Technology kit.

The cornerstone of the Infinity Technology kit is the TI VC-33 based board, the NI SPEEDY-33. Students use the board to learn about embedded signal processing and signal generation through various exercises included in the curriculum. The other major hardware components in the kit include a USB Webcam to perform image processing exercises such as building a blue screen, speakers for output. 
The NI SPEEDY-33 board (shown in figure 3) has one analog input and one analog output, two on-board microphones, 8 LEDs and 8 DIP switches and is used for all the audio processing exercises in the curriculum from the Infinity Project.

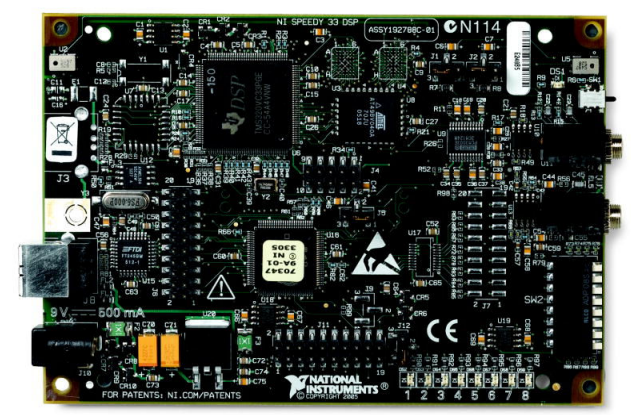

Figure 3 The NI SPEEDY-33(TI VC-33) DSP Starter Board used in the Infinity Technology Kit

It is well-known that programming a DSP is not trivial, even for embedded design engineers. However, here is a case where high-school students and teachers and freshmen engineering students get their applications running on the DSP board within one lab period. This is possible because the program uses the NI LabVIEW graphical programming environment, an industrystandard, which has been customized to suit the needs of the Infinity Project. The NI LabVIEW for the Infinity Project software is shown in figure 4.

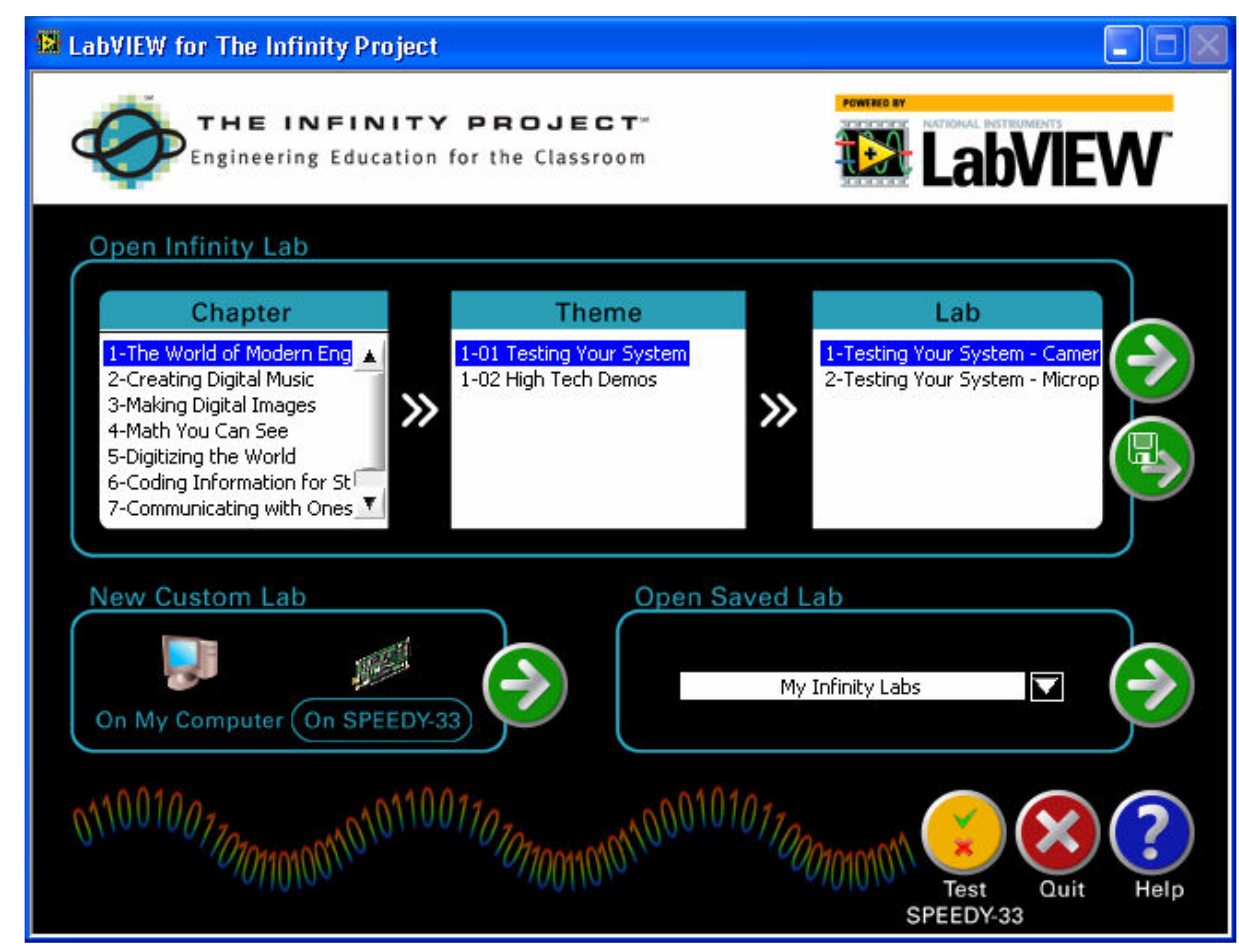

Figure 4. The NI LabVIEW for the Infinity Project Software Launch Screen

The launch screen shown in figure 4 has a lot of innovative features that enable teaching concepts. First, it has a lesson plan organizer that can be customized completely from the 
headings to the individual categories under each heading. The other innovative feature of the launch panel is the ability to create labs that run either on the computer (desktop or laptop) or on the SPEEDY-33 board. The reason for differentiating the two is because when the lab has to be developed for the board, it needs some specific input and output blocks that are not necessary when programming on the computer. Figure 5 shows the basic input and output blocks available for the SPEEDY-33. The key points worth noting are, first, there is no text-based script anywhere on the program, student program completely in blocks and second, the blocks are intuitively designed, for example, the different colors indicate the different data types. LEDs and DIP Switches are boolean (green color) and the analog input and output are numeric arrays (thick orange.

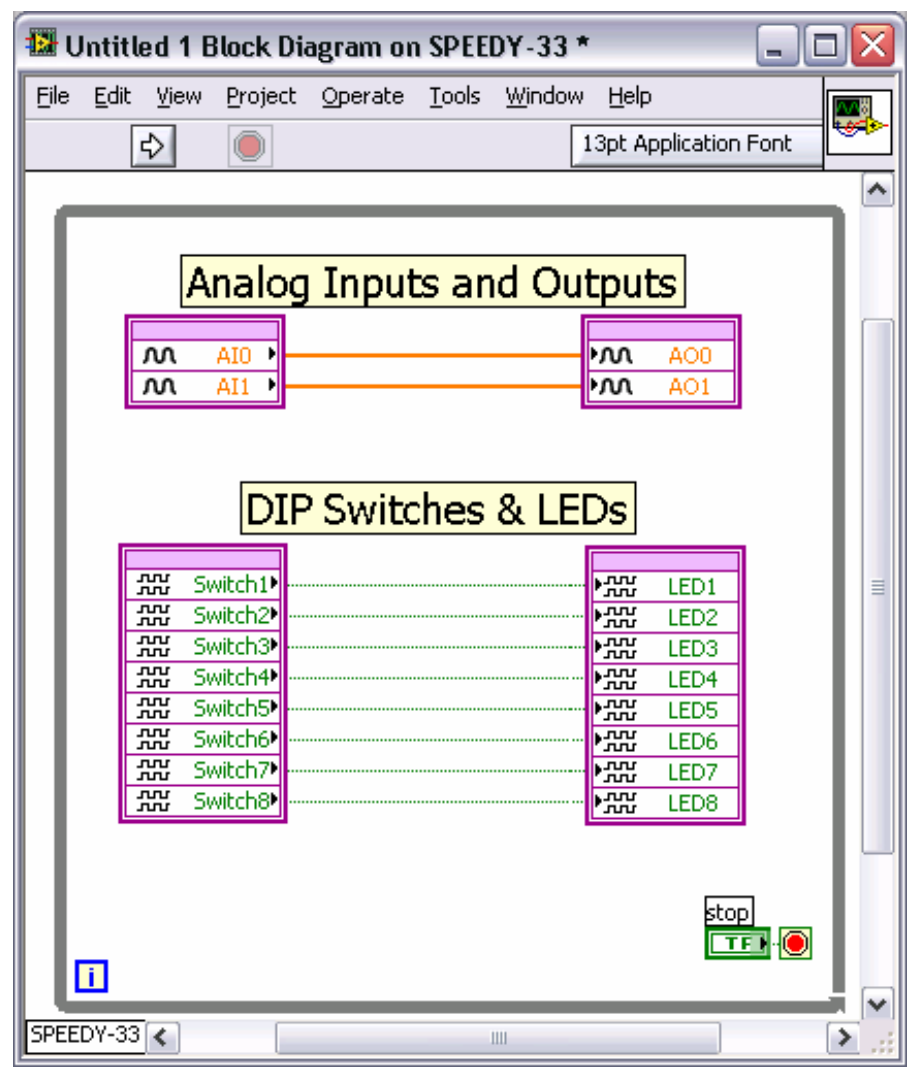

Figure 5. The I/O points available for the SPEEDY-33

Since the students are working in a graphical programming environment, it is also easier to communicate the errors. For example. Figure 6 shows an example where the incompatible datatypes are wired together. In the example shown, the student attempts to wire analog data to a block that accepts only boolean values. In this case, the software shows a broken wire with an " $x$ " between the connections. If the student hovers the mouse over the wire, he or she will get a message that says that he or she has wired two datatypes that are not compatible. In addition, the software prevents compiling the program if such errors are present - thus saving time and damage to hardware and avoid confusing the student. 


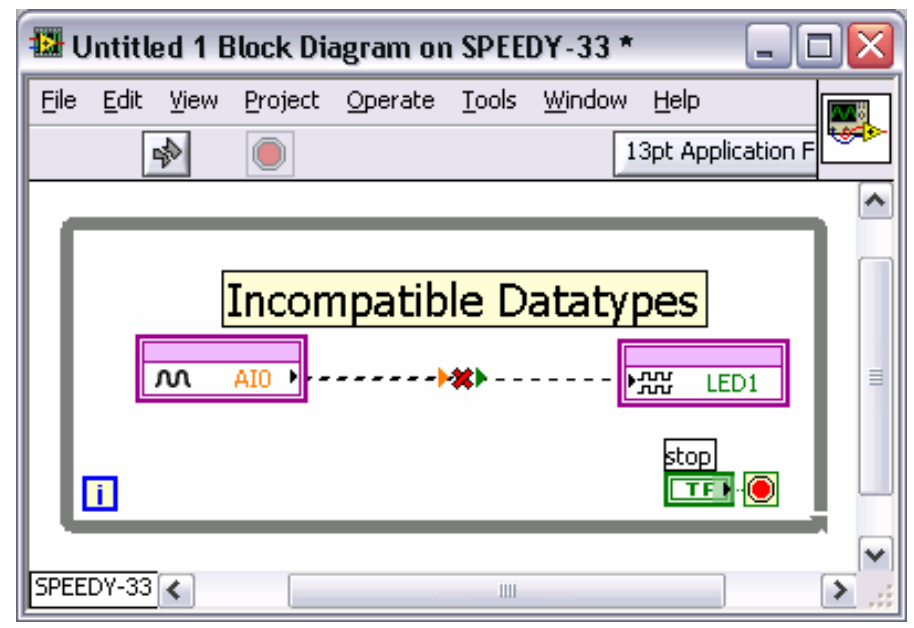

Figure 6. Intuitive Error Reporting Example

Figure 7 shows the way in which LabVIEW graphical programming enables students to target the VC-33 board.

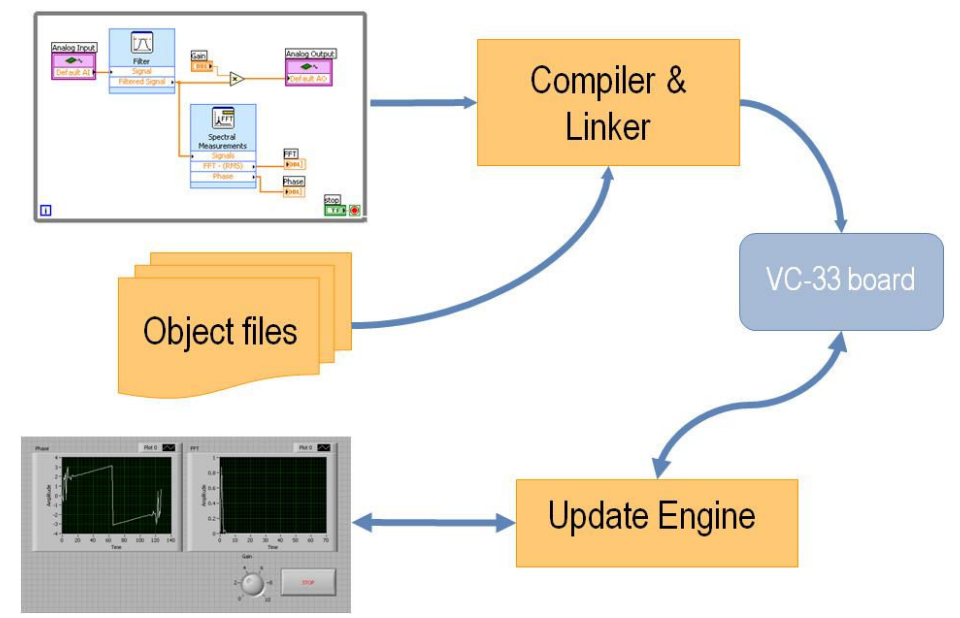

Figure 7. Targeting the SPEEDY-33 Board

To explain figure 7 in detail, for every block that is placed on the block diagram, there is an equivalent pre-compiled, pre-optimized binary available. When the students wire the blocks together, they are merely passing variables - this prevents code bloating. When the student presses the "Run" button, the LabVIEW engine compiles the primitives and links all the binaries together and downloads to target. Once the program is running on the target, then the user uses variables to poke into the registers of the board and display the data processed. The key is that all of the process after placing a block is seamless and hence makes it easy for the student to program the embedded board.

\subsection{Use Case: Extending Graphical Programming to Senior Design using FPGAs}

Let us know focus on an example where FPGA-based hardware and graphical programming has been used to teach embedded design. The mechatronics course at Rensselaer Polytechnic Institute is a multidisciplinary course between mechanical, electrical, computer and control engineering[11]. 


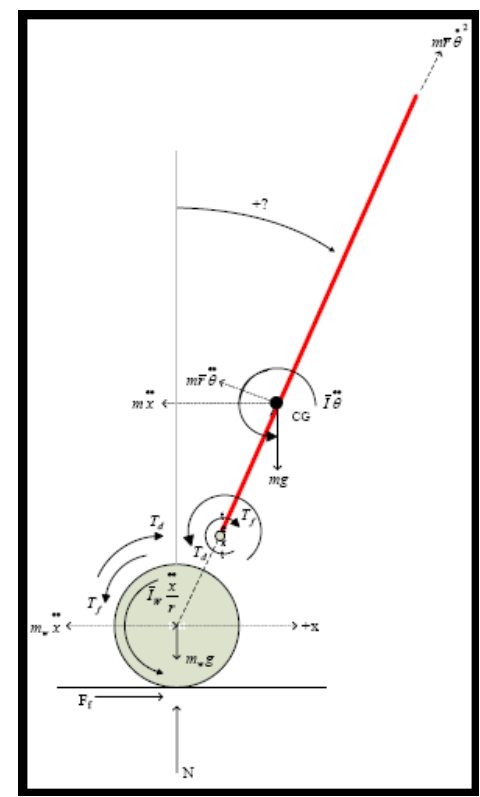

Figure 8. The Design Problem

Figure 8 shows the design problem that the students were tasked with. They had to build a Human Object Transport Vehicle (HOT-V) to improve human mobility and accessibility similar to the Segway ${ }^{\circledR}[12]$ systems. They had to build this system from design to prototyping and deployment in one semester. Because this project focused on design, prototyping and deployment, it helped teach students the concept of embedded design from ground-up. In order to meet the tight deadline on this project, the students chose to use NI LabVIEW, a graphical programming environment and designed their algorithms and tested it. Figure 9 shows a part of their simulation program.

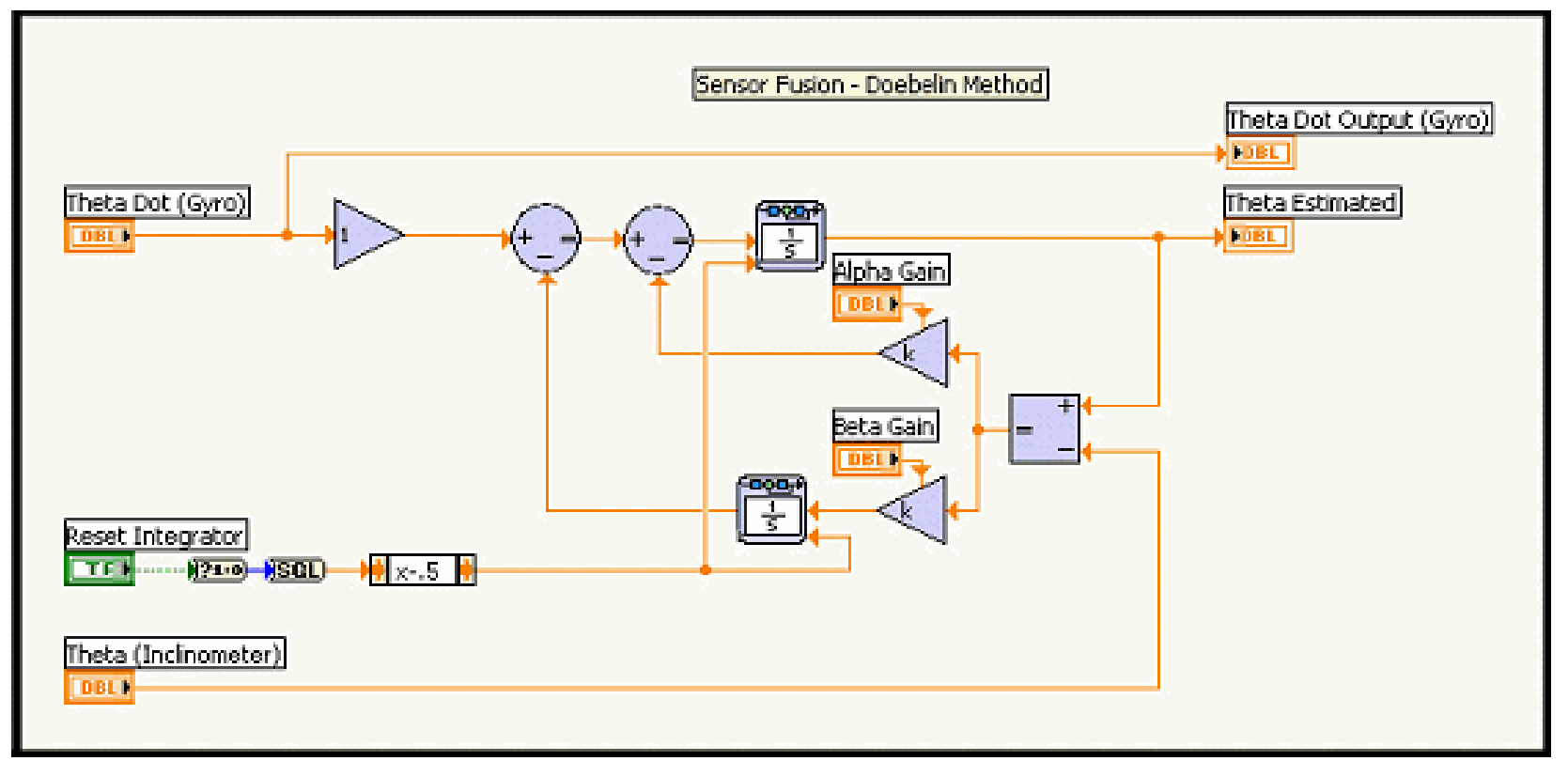

Figure 9. Example Code for the HOT-V 
The multidisciplinary group first prototyped their design on a tethered prototype that was connected to the computer as shown in figure 10.

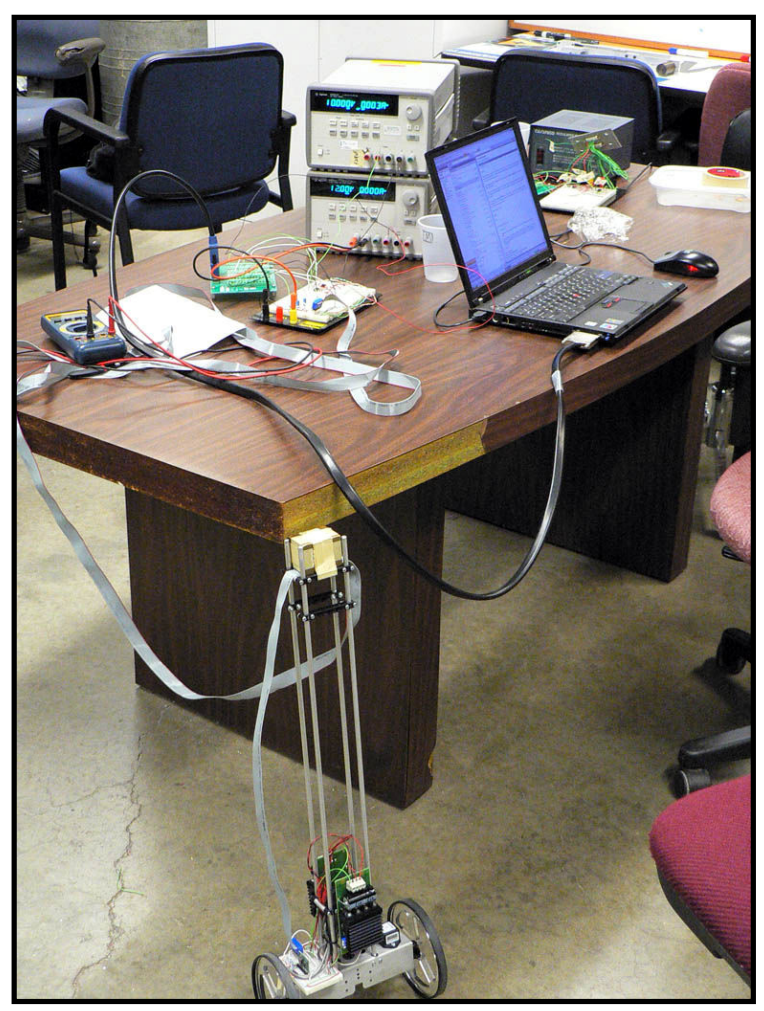

Figure 10. The First Prototype

After verifying that their simulation and design worked on the prototype, they built their actual HOT-V transport system. Key points to note here are that the students in this team were from multiple disciplines and the majority of them were not embedded programming experts. However, they were experts in their respective areas of control design, mechanical design, sensor interfacing.

The final design used NI CompactRIO - a hardware prototyping platform that is powered by an FPGA in the backplane and has a real-time operating system controlling the system. The FPGA and the real-time operating system can both be programmed graphically. Figure 11 shows the final version as a result of the project.
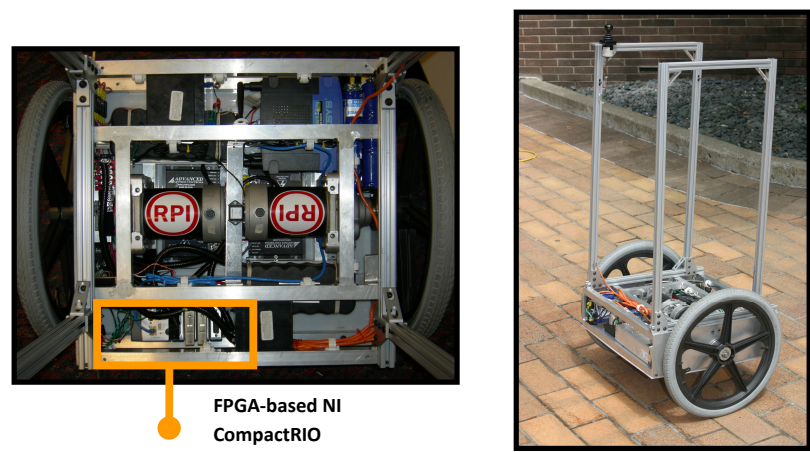

Figure 11. Final HOT-V Vehicle 
The students also went one step further and made the system controllable using a PDA and the time taken from start to finish for this project was half a semester. In the end, the students learnt the process of design flow, the process of testing and prototyping and completed the project successfully. This example shows the advantages of using Reconfigurable hardware and graphical programming to teach embedded design concepts.

\section{Conclusion}

The instruments and devices of the future will be embedded in some form or fashion. Thus, with our obligation to create excitement about the use of embedded devices early in engineering, we need innovative methodologies that can be used to teach with the embedded devices while maintaining a higher level of abstraction. Graphical programming provides a powerful solution for this problem, by helping abstract the implementation details at the same time providing a seamless path to targeting the embedded devices. In this paper, we illustrated this methodology with two use cases, a high-school and freshmen engineering program, The Infinity Project and a senior design project from RPI where the student build a human object transport vehicle in a semester from start to finish. Graphical programming has many other application areas from circuit design to communication systems and provides a flexible teaching solution for educators today.

\section{References}

[1] G Borriello, R Want, Embedded computation meets the World Wide Web - Communications of the ACM, 2000

[2] AH Taner, NM White, Virtual instrumentation: a solution to the problem of design complexity in intelligent instruments, Measurement+ Control, 1996

[3] Lee, E.A., “What's Ahead for Embedded Software”, Vol 33, Issue 9, IEEE Computer

[4] N. Halbwachs, P. Caspi, P. Raymond, and D. Pilaud. The synchronous data flow programming language lustre. Proceedings of the IEEE, 79(9):1305-1319, 1991.

[5] U. Banerjee, R. Eigenmann, A. Nicolau, and D. Padua. Automatic program parallelization Proceedings of the IEEE, 81(2):211-243, 1993.

[6] G. Berry. The effectiveness of synchronous languages for the development of safety-critical systems. White paper, Esterel Technologies, 2003

[7] WM Johnston, JRP Hanna, RJ Millar, “Advances in dataflow programming languages “, ACM Computing Surveys (CSUR), 2004

[8] The Ptolemy Project, http://ptolemy.eecs.berkeley.edu/

[9] Overview of LabVIEW, http://zone.ni.com/devzone/conceptd.nsf/webmain/F34045D2CC5357F486256D3400648C0F?O penDocument\&node=200067_us

[10] The Infinity Project, www.infinity-project.org 
[11] Mechatronics Course Website at RPI, http://mechatronics.rpi.edu/ [12] Official Segway Site, http://www.segway.com/

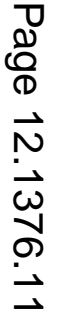

